\title{
LA DIVULGACIÓN DE «EL FIN DE LA HISTORIA» EN ESPAÑA
}

\author{
Por \\ ISRAEL SANMARTÍN ${ }^{1}$
}

El mundo intelectual estadounidense ha disfrutado en los últimos años de intensos debates alrededor de diferentes obras. Primero han sido los libros de Paul Kennedy The rise and fall of the great powers: economic change and military conflict from 1500 to $2000^{2}$ y Allan Bloom The closing of the american mind ${ }^{3}$; y más tarde fueron los ensayos de Francis Fukuyama The end of History and the last man $^{4}$ y Samuel Huntington The clash of civilitations and the remaking of world order ${ }^{5}$. Estos dos últimos han sido dados a conocer con una misma estiategia. Primero se publicaba un artículo polémico y provocador con una promoción digna de cualquier estreno cinematográfico. Después, la misma revista que editaba el artículo promovía replicas de varios críticos. Finalmente, el responsable del

\footnotetext{
${ }^{1}$ Este trabajo está realizado dentro del marco del Proyecto de Investigación «El estado de la historia», dirigido por el Dr. Carlos Barros en el Instituto de Estudios Gallegos «Padre Sarmiento»(CSIC) y financiado por la Xunta de Galicia (XUGA 40101B96)

${ }^{2}$ KENNEDY, Paul, The rise and fall of the great powers: economic change and military conflict from 1500 to 2000, New York, 1987.

${ }^{3}$ BLOOM, Allan, The closing of the american mind, Simon and Schuster, New York, 1987.

${ }^{4}$ FUKUYAMA, Francis, The end of History and the last man, The Free Press, New York, 1992.

${ }^{5}$ HUNTINGTON, Samuel, The clash of civilitations and the remaking of world order, Simon and Schuster, New York, 1996.
}

"CUADERNOS DE ESTUDIOS GALLEGOS", Tomo XLVII, Fascículo 113, Santiago 2000. 
ensayo escribía un libro para contestar a sus críticos y ampliar los argumentos de su primer trabajo ${ }^{6}$.

En este trabajo se analiza la divulgación ${ }^{7}$ de la tesis de «el fin de la Historia» de Francis Fukuyama ${ }^{8}$ en España. Se publicaron tres $^{9}$ de los cinco trabajos que componen el total de la tesis. Los trabajos traducidos al castellano fueron los artículos «The end of History?» $\mathrm{y}$ «A reply to my critics» y el libro «The end of History and the last man». Las traduciones de los artículos se realizaron gracias a dos publicaciones del grupo de comunicación PRISA: El País y Claves de Razón Práctica, aunque también el diario $A B C$ difundió muy reducidamente el artículo «¿el fin de la Historia?». Por otro lado, el libro The end of History and the last man fue volcado al castellano gracias a la editorial Planeta.

\footnotetext{
${ }^{6}$ Recientemente ha aparecido un caso de las mismas características. Alan Sokal (físico de la Universidad de New York) escribió un artículo sin sentido y lo envió a la revista Social Text, donde incluso le hicieron un número monográfico para celebrar su publicación. Una vez publicado, Sokal declaró que todo era una broma y que su publicación constataba el relativismo actual de la ciencia, donde a menudo se emplean términos y acepciones sin sentido y desde la ignorancia. Ahora, acaba de publicar un libro con el belga Jean Bricmont (Fashionable nonsense: postmodern intellecuals abuse of science, Picador, 1998), donde ataca a los intelectuales franceses postmodernos. Estos se defienden argumentando que se trata de una guerra cultural y política, no intelecual. Ver, ROTHSTEIN, Edward, «Batalla entre físicos y metafísicos», El País, 5 enero 1999, p. 23. Y también: http://www.larecherche.fr/FOR/SOKAL/, http://zakuro.math.tohoku.ac.jp/ $\sim$ kuroki/Sokal/history-j.html, http://www.physics.nyu.edu/faculty/sokal.html, http:// www.liberation.fr/forums/sokal/.

${ }^{7}$ Para que no haya dudas del objetivo del trabajo, según el diccionario de la lengua de la Real Academia española la palabra «divulgar» significa: «Publicar, extender, poner al alcance del público una cosa». Ver, Voz «Divulgar» en Diccionario de la Lengua Española, Real Academia Española, Madrid, 1984, Tomo I, p.510. También significa: «Difundir. Generalizar. Propagar. Hacer llegar cierto conocimiento al vulgo o a las personas ajenas al campo a que corresponde específicamente ese conocimiento» y «poner al alcance de la generalidad de la gente algo que antes estaba reservado a una minoría». Ver voz «Divulgar» en MOLINER, María, Diccionario de uso del español, Editorial Gredos, Madrid 1992, p. 1026.

${ }^{8}$ Para una mayor profundización en el autor, ver: SANMARTÍN, Israel, «QQuién es Francis Fukuyama?», Cuadernos de Estudios Gallegos, Tomo XLVI, Fascículo III, 1999, pp. 193-206.

${ }^{9}$ En el momento de entregar este trabajo se ha publicado el libro Trust en ediciones B.
}

"CUADERNOS DE ESTUDIOS GALLEGOS", Tomo XLVII, Fascículo 113, Santiago 2000. 
El artículo sobre «el fin de la Historia $»^{10}$ de Francis Fukuyama («The end of History?»), fue publicado originalmente en la revista norteamericana de pensamiento político y relaciones internacionales The National Interest en el verano de 1989. En esa primera publicación, usaba cinco subcapítulos para demostrar la tradición hegeliana de la teoría de «el fin de la Historia», constatar "el fin de la Historia» en el actual sistema demócrata liberal, y afirmar la muerte del marxismo-leninismo. La divulgación del trabajo se adelantó unos meses a la caída del muro del Berlín, haciendo parecer a Fukuyama como un futurólogo. Sin duda, esta coincidencia, junto con una labor propagandística espectacular, hicieron que el ensayo se llegara a debatir y conocer en muchos círculos intelectuales de todo el mundo.

Debido a la gran repercusión del artículo rápidamente se tradujo al castellano, y como en otros muchos países, el artículo «The end of History?» se divulgó mediante la prensa diaria. Esa labor correspondió en España a los diarios El País y $A B C$ en un principio y posteriormente a la revista de pensamiento Claves de Razón Práctica. La traslación del diario El Pais fue muy utilizada por los comentaristas de Fukuyama, pero pocos se dieron cuenta que era una traducción que no llegaba a la mitad del original. A esta conclusión sólo se puede llegar analizando el texto cualitativa y cuantitativamente y observando cuánto y qué falta en la traducción respecto al original. La versión de Claves de Razón Práctica era más completa pero ofrecía erratas y olvidos considerables que son apuntados más adelante.

La gran resonancia y las grandes críticas recibidas por su primer artículo hicieron que Fukuyama publicara un segundo artículo de contestación. El trabajo se tituló «A reply to my critics» y se editó dos números después en la misma The National Interest. En esta nueva publicación

${ }^{10} \mathrm{El}$ término «Historia» aparecerá con mayúsculas en el resto del trabajo cuando se refiera a la teoría de «el fin de la Historia». Fukuyama alude a la Historia (con «H» mayúscula) como un proceso único, evolutivo y coherente de todos los pueblos en todos los tiempos (es la Historia entendida partiendo del concepto elaborado por Hegel y Marx). Sin embargo, no menciona, en el título de su tesis, a la historia (con «h» minúscula), entendida como la mera sucesión de acontecimientos. En las diferentes reproducciones de críticos y comentaristas se respetará el criterio de los mismos. Aunque hay otros significados del térmico «Historia» como el que se refiere a la disciplina científica.

"CUADERNOS DE ESTUDIOS GALLEGOS", Tomo XLVII, Fascículo 113, Santiago 2000. 
aclaraba el concepto de «Historia» en Hegel; explicaba la poca trascendencia que tenía, a su juicio, su pertenencia a la Administración Bush; y respondía a los argumentos sostenidos por muchos de sus críticos en relación a las ideologías alternativas a la democracia liberal. También esta segunda publicación fue traducida al castellano por el diario El País con título «Respuesta a mis críticos». Esta vez fue difundida, con más atino, íntegramente, y al poco tiempo de la original.

Como «A reply to my critics» no había solucionado nada, el norteamericano abandonó su puesto de funcionario en laAdministración Bush y se dedicó a escribir el libro The end of History and the last man ${ }^{11}$ publicado en 1992 e inmediatamente traducido a múltiples idiomas. El libro era una ampliación teórica y empírica de su primer ensayo. Constaba de cinco capítulos donde desarrollaba dos Historias Universales. Una basada en la «ciencia natural moderna» (y, sobre todo, en Marx), que correspondía a una explicación económica de la Historia donde emparentaba la Historia con el desarrollo tecnológico. Y otra cimentada en el «deseo de reconocimiento» (y, sobre todo, en Hegel), que incumbía a una interpretación antropológica de la Historia que la unía con la democracia liberal y con el final de la Historia, al no existir ninguna organización política superior a la democracia liberal, después de caer el fascismo y el comunismo.

¿Cómo elabora Fukuyama esas historias universales? Descartando pueblos enteros, sobre todo los prehistóricos, y aparcando los países que no llegaron a «el fin de la Historia», o sea, los no históricos (en contraposición a los poshistóricos -que han llegado al fin de la Historia-). Utiliza una metodología de exclusión que le hace construir unas Historias Universales occidentalistas, basadas más bien en EE. UU. que en Europa, obviando los países de África, gran parte de Asia y algunos sudamericanos. De este modo, Fukuyama coincidía con las premisas de Historia Universal del siglo XIX, donde se tomaba como tal la que después de las etapas previas de Egipto y Mesopotamia, concernía a Grecia y Palestina hasta nuestros momentos. El resto pertenecería a la etnología y quedaría

\footnotetext{
"Traducción española: FUKUYAMA, Francis, El fin de la Historia y el último hombre, trad. P. Elías, Planeta, Barcelona, 1992.
}

"CUADERnOS DE ESTUdiOS GALLEGOS", Tomo XLVII, Fascículo 113, Santiago 2000. 
fuera de la verdadera historia. Así la Historia Universal era, para Fukuyama, la Historia de Occidente ${ }^{12}$.

Por otro lado, en 1994, gracias al profesor Timothy Burns en el libro After History? Francis Fukuyama and his critics $^{13}$, Fukuyama tuvo la oportunidad de dar una última contestación a los numerosos exégetas de su tesis, aunque se quedó en una simple réplica a los comentarios de una docena de profesores universitarios, en su mayoría norteamericanos. El artículo se titulaba «Reflections on the end of History, five years later» ${ }^{14}$. En esa ocasión presentaba su tesis de una forma novedosa, desglosándola en «empírica» y «teórica». Explicaba cada división por separado y las relaciones entre ambas. Este trabajo no tiene una traducción publicada en castellano ${ }^{15}$.

Y en 1995 publicó Trust. the social virtues and the creation of prosperity ${ }^{16}$, un desarrollo de uno de los aspectos tratados en El final de la Historia y el último hombre: el asociacionismo encarnado en la sociedad civil. En este último libro Fukuyama reconoce la importante labor del Estado en el desarrollo económico de un país y analiza la importancia de la cultura en la economía.

\section{LA DIVULGACIÓN RESUMIDA DE «¿EL FIN DE LA HISTO- RIA?» EN ESPAÑA}

El artículo de Fukuyama desató también una importante polémica en España. Debido a que la revista The National Interest no se distribuye en la Península Ibérica, la difusión del artículo «The end of History?» co-

12 JASPERS, Karl, Origen y meta de la historia, trad. Fernando Vela, Alianza editorial Madrid, 1980, p. 16 (Madrid, 1950).

${ }^{13}$ BURNS, Timothy (ed.), After History? Francis Fukuyama and his critics, Rowman \& Littlefield Publishers, Maryland, 1994.

${ }^{14}$ FUKUYAMA, F., «Reflections on the end of History, five years later» en Timothy Burns (ed.), After History? Francis Fukuyama and his critics, pp. 239-258. También reproducido en History and Theory, vol. 34, 1995, pp. 27-43.

${ }^{15}$ Se puede encontrar una traducción en SANMARTíN, Israel, La Historia según Francis Fukuyama, 1989-1996, Tesis de Licenciatura, Santiago de Compostela, 1996

${ }^{16}$ FUKUYAMA, F., Trust: the social virtues and the creation of prosperity, New York, 1995; En el momento de la entrega de este trabajo ha sido traducido al español como: La Confianza, Ediciones B, 1998.

"CUADERNOS DE ESTUDIOS GALLEGOS", Tomo XLVII, Fascículo 113, Santiago 2000. 
rrespondió al diario $A B C$ y a dos publicaciones del grupo de comunicación PRISA ${ }^{17}$, una de las empresas periodísticas más importantes de España y de Europa. Los dos medios que divulgaron el ensayo fueron la revista Claves de Razón Práctica ${ }^{18}$ y el diario El País ${ }^{19}$. En ambos casos se trataba de un resumen extractado del original. A ellas acudieron todos los críticos españoles del artículo, con lo que se quedaron sin conocer gran parte del trabajo. Aunque la idea general estaba bien reflejada en las traducciones, sus opiniones estuvieron condicionadas por la falta de algunos datos. En este sentido, el director del Centro de Estudios de Política Exterior-Santiago de Mora-Figueroa-, escribía:

«Dudo que mucha gente haya podido leerlo. Además la moda de Fukuyama se extendió en el acto a Europa, donde es aún más dificil hacerse con el texto completo. En España, por ejemplo, no creo que ningún comentarista lo conozca; todos parecen guiarse por la versión publicada en El País (24-9-89). Esta última es un resumen cuatro veces más corto que el original. Tal mutilación no es en sí un sacrilegio: el mensaje de Fukuyama es tan sencillo que puede extractarse más aún ${ }^{20}$.

El resumen no era cuatro veces más corto que el original sino algo más de la mitad, como se verá más adelante, pero en ningún caso se debe hacer la crítica de una argumentación sin conocerla en su totalidad, y menos extractándola todavía más, como pretendía Mora-Figueroa. De todos modos, los editores del diario El País tuvieron una indudable responsabilidad en la proyección del artículo de esta forma (suprimieron las citas a pie

\footnotetext{
${ }^{17}$ El grupo Prisa engloba radios (Cadena Ser, 40 Principales, M-80, Sinfo Radio y Cadena Dial), televisión (Canal +, Canales digitales), revistas (Cinemanía, El País Semanal o Claves de razón práctica), prensa diaria (Cinco Días, As y El País) y actividad editorial (Anagrama, Santillana o Aguilar). Esta empresa periodística mantiene una cierta preocupación cultural en sus publicaciones e intenta reflejar en sus distintos suplementos y revistas especializadas, las últimas tendencias intelectuales y culturales. Los medios elegidos por el grupo PRISA para la divulgación del ensayo ¿El final de la Historia? fueron el diario El País y la revista Claves de razón práctica.

${ }^{18}$ FUKUYAMA, Francis, «¿El final de la Historia?», Claves de Razón Práctica, Madrid, $n^{\circ}$ 1, Abril 1990, pp. 85- 96.

${ }^{19}$ FUKUYAMA, Francis, «¿El final de la Historia?», El País, Madrid, 24 de septiembre, 1989.

${ }^{20}$ MORA FIGUEROA, Santiago de, «El acabose», Nueva Revista, febrero 90, n 1, p. 41.
}

"CUADERNOS DE ESTUDIOS GALLEGOS", Tomo XLVII, Fascículo 113, Santiago 2000. 
de página, obviaron muchas partes del artículo, había algunos errores de traducción y añadieron una entradilla al artículo). Los editores del periódico más leído y vendido de España, sólo cumplieron con la misión de dar a conocer, de un modo masivo, la famosa teoría de «el fin de la Historia». No estaba en el ánimo de los dirigentes del rotativo madrileño una utilización científica del trabajo. En todo caso, sería problema de los críticos buscar la versión original para comentarla.

La traducción de la revista Claves de Razón Práctica fue publicada en el número inicial de esa publicación periódica cuatro meses después de la del diario El País. Fue una traslación al castellano mucho más completa, incluía las citas a pie de página y reunía un total de doce páginas. A pesar de todo, Agustín García Calvo juzgaba la traducción como «poco satisfactoria $»^{21}$, cinco números después, en la misma revista.

La difusión del artículo en el diario $A B C$ era más interpretativa y volcada más hacia el debate. En ningún momento pretendía ofrecer el texto original, sólo algunas ideas que contenía. En los siguientes subapartados se abordará el tema de las traducciones más en profundidad.

\section{La traducción de El País. Septiembre de 1989}

La traducción de El País fue el texto más común en las citas de los diferentes críticos y para el debate sostenido en la prensa durante varios meses $^{22}$. En su presentación, constaba de dos páginas completas del perió-

${ }^{21}$ GARCÍA CALVO, Agustín, «Tras el fin de la Historia», Claves de Razón Práctica, octubre $1990, \mathrm{n}^{\circ} 6$, p. 11.

${ }^{22}$ Para el particular véase: LEIGHT, Robert, «El abrazo de Alemania», El País, 16 de noviembre de 1989; VÁZQUEZ MONTALBÁN, M., "Historia», El País, 4 septiembre 1990; TALBOTT, Strobe, «El principio del absurdo», El Pais, 30 de septiembre 1989; TOURAINE, Alain, «La caída del segundo muro», El País, 11 de noviembre, de 1989; HOBSBAWM, E., «1989: para el vencedor, los despojos», El País, 23 de octubre 1990; GIL CALVO, Enrique, «La apertura del legado de Marx», El País, 9 de enero de 1990, p. 11; BARRANCO, Juan, «Fukuyama versus Erich Fromm», El País, 28 de mayo de 1990, p. 42; BARRANCO, Juan, «Del pesimismo de Fukuyama y otros pesimismo anteriores», EL País, martes 29 de mayo de 1990, p. 44; NEGRI, T., «Fin de siglo», El Mundo, 1 de abril 1990; USLAR PIETRI, Arturo, «El triunfo de occidente y las erróneas profecías sobre el fin de la historia», La Voz de Galicia, 13 de noviembre de 1989; AYALA, Francisco, «Postrimerias de la historia», La Voz de Galicia, 25 de octubre de 1990; JIMÉNEZ DE PARGA, Manuel, «Estalló la historia», Diario 16 de Galicia, 20 agosto 1990; COSTA, X. M., "O fin da historia», La Voz de Galicia, 5 de noviembre 1990; CANCIO, M., «El comunismo estalinista», La Voz de Galicia, 17 de noviembre de 1989.

"CUADERNOS DE ESTUDIOS GALLEGOS", Tomo XLVII, Fascículo 113, Santiago 2000. 
dico. En la primera hoja incluía una ilustración de las cabezas de Hegel y Marx con la frase «The end» en el medio. En la segunda incluía una foto de Churchill, Roosevelt y Stalin durante la conferencia de Yalta. En el pie de texto de la ilustración de Marx y Hegel se podía leer The Independent/ El País, lo que podría hacer pensar que la traducción al español había sido hecha sobre un extracto previamente realizado por el periódico británico progresista The Independent.

De todas formas, el original de The National Interest salió a la venta en el mes de junio de 1989 y la traducción de El País se publicó el 24 de septiembre de 1989. Por lo tanto, tuvo un retraso de, aproximadamente, tres meses ${ }^{23}$. A pesar de la diferencia de fechas el texto de El País alcanzó una significancia especial.

Cambiando de tercio, para este particular de la investigación, se escogerá la técnica del Análisis de contenido ${ }^{24}$ tanto en su variante cualitativa como cuantitativa. Debido a esto se analizará el texto, en referencia al original, tanto cualitativa como cuantitativamente. El estudio cuantitativo se refiere a cuánto y qué se tradujo; el cualitativo a la relevancia, importancia y trascendencia de lo que se tradujo ${ }^{25}$. Sobre las técnicas del Análisis de contenido se ha escogido el método de análisis sistemático ${ }^{26}$, to-

${ }^{23}$ En ese tiempo algunos intelectuales hacen algunos comentarios en prensa, como: MENDO, Carlos, «El fin de la historia», El Pais, 31 de agosto, 1989.

${ }^{24}$ El Análisis de contenido es una técnica antigua, pero desarrollada hoy sobre bases mucho más sofisticadas, que resulta esencial en el análisis cualitativo de datos. Se trata de una técnica basada en el análisis del lenguaje, pero cuyo objetivo no es conocer éste en sí mismo sino «inferir» alguna otra realidad distinta a través de él. El análisis de contenido empezó como análisis de la propaganda y del lenguaje político; (ARÓSTEGUI 1995: 370). Aróstegui define la técnica del Análisis de contenido en base a BARDIN, L. Análisis de contenido, Akal, Madrid, 1986; como: «un conjunto de técnicas de análisis de las comunicaciones tendente a obtener indicadores (cuantitativos o no) por procedimientos sistemáticos y objetivos de descripción del contenido de los mensajes, permitiendo la inferencia de conocimientos relativos a las condiciones de producción/ recepción (variables inferidas) de estos mensajes» (ARÓSTEGUI 1995: 370).

${ }^{25}$ Uno de los objetivos del trabajo es combinar, en la medida de las posibilidades y tipo de trabajo, lo cuantitativo y lo cualitativo, tal y como señala BARROS, Carlos, Mentalidad y revuelta, Tesis doctoral, ed. facsímil, Santiago de Compostela, 1988.

${ }^{26}$ Clasificación realizada en base a la mostrada por ARÓSTEGUI, J., La investigación histórica: teoría y método, pp. 358-398, aunque se ha tenido en cuenta que «Las técnicas del Análisis de contenido son siempre muy abiertas. Se ha dicho que es preciso inventarlas cada vez que se emplean» (ARÓSTEGUI 1995: 370). Por esa razón, las clasificaciones hechas en el trabajo no se ajustan estrictamente a las dadas por Aróstegui.

"CUADERNOS DE ESTUDIOS GALLEGOS", Tomo XLVII, Fascículo 113, Santiago 2000. 
mando para el análisis cuantitativo el párrafo como unidad básica de codificación, y para el examen cualitativo un examen del contenido temático de cada uno de los epígrafes del texto.

\section{Análisis cuantitativo del extracto del diario El País}

El análisis cuantitativo es necesario en el sentido de dejar claro y en números qué es realmente lo que se tradujo en aquel texto, y qué se obvió. El método cuantitativo es el mejor para reflejar esos resultados ${ }^{27}$. Sin embargo, no se debe olvidar el tratamiento cualitativo de la información: el estudio cuantitativo de la traducción dará los resultados numéricos pero no la importancia y relevancia de los párrafos traducidos.

El volumen de texto que englobaba la traducción no llegaba a la mitad del original, concretamente alcanzaba el $44.8 \%$. El número de páginas era dos; por dieciséis del original. El número de párrafos ${ }^{28}$ de la versión de $E l$ País se quedaba en veintitrés frente a los cincuenta y ocho del difundido por la revista National Interest ${ }^{29}$ (ver tabla 1). En otro sentido, en el texto

${ }^{27}$ Aunque se trata de un trabajo que trata de unir la Historiografía con la Teoría de la Historia, no significa que el método cuantitativo no tenga cabida. Siempre que se pueda se debe cuantificar y no abandonar el rigor crítico y documental; lo que Carlos Barros llama positivismo «bueno», como un paso obligado para entrar y desarrollar en el siglo XXI historiográfico. BARROS, Carlos, «La Historia que viene», Historia a Debate, tomo I, Santiago de Compostela, 1995, p. 116. También en BARROS, Carlos, Mentalidad y Revuelta, Tesis doctoral, ed. facsímil, Santiago, 1988, p. 17, allí consideraba que «aparte del rigor metodólogico que aporta, la investigación con base cuantitativa permite descubrir realidades ocultas para un tratamiento cualitativo simple». En la página siguiente añadía: «estos fogonazos empíricos causan a veces la sorpresa del investigador, que en más de una ocasión tiene que rectificar para sí una previa opinión impresionista, basada en tal o cual dato pero no en la totalidad de los conocidos y utilizados para la cuantificación: así es como se aprende en carne propia a valorar el método cuantitativo». Estas afirmaciones estaban realizadas para apuntalar el estudio de los Irmandiños, pero son igualmente válidas para este caso. Se trata de mostrar y captar la verdadera realidad de la traducción y no cometer errores de cálculo como el de Santiago de Mora-Figueroa.

${ }^{28}$ Entendiendo como párrafo: «Cada una de las divisiones de un escrito señaladas por letra mayúscula al principio del renglón y punto y aparte al final del trozo de escritura»; Diccionario de la lengua española de la Real Academia Española, tomo II, 21 edición, Madrid, 1994.

${ }^{29}$ En este caso, el número de párrafos hace referencia al aspecto formal del texto tal y como aparece en cada medio. Los párrafos no tienen correspondencia entre ambos textos. Es una referencia descriptiva del texto.

"CUADERNOS DE ESTUdIOS GALLEGOS", Tomo XLVII, Fascículo 113, Santiago 2000. 
en castellano no aparecía ninguna de las diecinueve notas del original y de los cinco epígrafes del ensayo original únicamente aparecían cuatro, y sin ninguna correspondencia entre sí. Los epígrafes del original eran numéricos e iban del «I» al «V»; los rótulos de la traducción eran: «El último paso», «Pérdida de atractivo», «Formas distintas» y «violencia étnica». Otra de las características de la traducción incumbía al título, presentaba «historia»con «h» minúscula en comparación a la mayúscula del original de Fukuyama (ver tabla I).

En otro orden de cosas, la entradilla que antecedía al texto era una introducción, con la función de presentación, que no se correspondía con la primera versión. La entradilla rezaba:

"¿Una teoría para concluir con todas las teorías? Esto es lo que parece pretender el provocativo artículo de Francis Fukuyama, número dos de la oficina de planificación a largo plazo del Departamento de Estado norteamericano. El artículo que ofrecemos extractado... El final de la historia que describe se ha convertido en la nueva Biblia de la derecha norteamericana, ha originado una fuerte polémica en Estados Unidos y es la última aportación al debate abierto tras el fin de la guerra fría ${ }^{30}$.

${ }^{30}$ FUKUYAMA, Francis, «¿El fin de la historia?», El País, p. 10. En líneas generales el juicio de valor expresado en la entradilla del texto era cierto, sin embargo, Joseph, McCarney explicaba tres años después que parte de la derecha norteamericana había rechazado los postulados de Fukuyama. La razón residía en que Fukuyama utilizaba una interpretación marxista de la Historia para llegar a una conclusión no marxista. McCARNEY, Joseph, «Endgame», Radical Philosophy, 62, Autumn, 1992, p. 35. También desde posiciones conservadoras, el director de la revista The National Interest aseguraba, en referencia al supuesto final de la Historia, que «No me creo una palabra de todo, aunque... el brillante análisis de Fukuyama no es fácil de rechazar»; en KRISTOL, Irving, «Response to Fukuyama», The National Interest, $\mathrm{n}^{\circ} 16$, summer 1989, p. 27. Tampoco desde la derecha española, en la persona de José María Carrascal, fue bien recibido Fukuyama, en el $A B C, 7,12,89$. Perry ANDERSON, desde posturas progresistas, consideraba el «marxismo invertido» de Fukuyama como un tributo a la izquierda, en ANDERSON, Perry, A zone of Engagement, Verso, London- New York, 1992.

"CUADERNOS DE ESTUDIOS GALLEGOS", Tomo XLVII, Fascículo 113, Santiago 2000. 
Tabla 1. Aspecto formal del texto traducido en relación al original

\begin{tabular}{lcc}
\hline & Original (The N. Interest) & Traducción española: El País \\
\hline No de páginas. & 16 & 2 \\
$\mathrm{~N}^{\circ}$ de párrafos. & 58 & 23 \\
$\mathrm{~N}^{\circ}$ de notas a pie de & & \\
página (incluyendo el & 19 & 0 \\
agradecimiento inicial). & 5 & 4 \\
No de epígrafes del texto. & & «h» minúscula \\
La palabra «Historia» en & «H» mayúscula & \\
el título del artículo. & & \\
\hline
\end{tabular}

Finalizado el aspecto formal de la traducción, el siguiente paso es examinar el número de párrafos vertidos al castellano y sus porcentajes en relación a la versión original. El número total de párrafos trasladados al español fue de veintiséis, que simbolizaba el $44.8 \%$ de los cincuenta y ocho del original. De estos veintiséis párrafos quince aparecían traducidos en su totalidad (un 27,6\%) y once parcialmente (el 18.9\%). Con estos datos se demuestra el escaso número de párrafos traducidos íntegramente y la gran cantidad de ellos, treinta y dos, que quedaron inéditos.

Todos estos números significan que la versión del texto de «¿El fin de la Historia?» aparecido en El País, que fue el medio mediante el cual muchos de los críticos de Fukuyama conocieron la teoría, era un resumen de menos de la mitad del original, considerando como unidad los párrafos. Del mismo modo se obviaban los pies de páginas y había múltiples incorrecciones y algún añadido (como la entradilla).

Del desglosamiento en párrafos, se desprende que un $55.2 \%$ no fue traducido. Sólo se tradujeron 26 párrafos y únicamente 15 en su totalidad. ¿Que quiere decir esto? Que los traductores se dedicaron a hacer un refrito del texto recogiendo retales de un lado y de otro para destacar lo sustantivo y lo llamativo del texto, a su juicio. El criterio de los traductores se limitó a copiar la introducción y el último capítulo, a obviar los dos primeros epígrafes y a hacer un «collage» de los epígrafes III y IV.

"CUADERNOS DE ESTUDIOS GALLEGOS", Tomo XLVII, Fascículo 113, Santiago 2000. 
En cuanto a los párrafos traducidos parcialmente, mediante la tabla 4 se puede observar que más de la mitad (en concreto seis) no eran traducidos ni al 50\%. Esto nos indica el nivel ínfimo de la traducción del artículo.

En este apartado se pretende convertir en medibles circunstancias que son difíciles de observar mediante una simple lectura del texto y a descubrir nuevas lecturas del mismo mediante los números, sin alterar las condiciones cualitarivas del mismo, pese a que la cuantificación es mirada bajo sospecha hoy en día ${ }^{31}$. Se trata de intentar reflejar las relaciones exactas entre lo traducido y el original a sabiendas que cuantificar no es un fin en sí mismo ${ }^{32}$.

\section{Análisis cualitativo del extracto del diario El País}

El análisis cualitativo ${ }^{33}$ del diario El País, complementa al examen cuantitativo realizado en el apartado anterior. Para ello se analizará el contenido de cada epígrafe del texto «¿El final de la Historia?». Siempre teniendo en cuenta que el análisis cualitativo describe las variables en un

${ }^{31}$ ARÓSTEGUI, Julio, La investigación histórica: teoría y método, Crítica, Barcelona, 1995, p.380.

${ }^{32}$ ARÓSTEGUI, J., La investigación histórica: teoría y método, p. 382; donde, además, señala que «la investigación cuantificada tiene los mismos fines que la cualitativa. La cuantificación permite encontrar relaciones, explicaciones de comportamientos, que muchas veces permanecen ocultas a una investigación cualitativa». Por otro lado, Aróstegui (pp. 381-382) señala que «la cuantificación cumple, al menos, tres importantes funciones metodológicas: 1- obliga a expresar claramente los presupuestos de los que se parte, a desarrollar con especial precisión los argumentos y procura una mayor facilidad de refutación de lo que se expone. 2- La presentación conjunta de los datos cuantitativos hace más probable que la aparición de casos no contemplados o inusuales sea mejor detectada, al tiempo que sea más fácil la observación del comportamiento a lo largo de diferentes períodos, grupos o espacios de alguna cualidad. 3- El empleo del lenguaje matemático y la presentación de los datos de forma ordenada hace más factible que otros investigadores comprueben, verifiquen, o refuten las conclusiones establecidas».

${ }^{33}$ «El tratamiento estadístico de la información histórica revela tendencias, proporciones, diferencias, cambios, discontinuidades,.... pero no explica sus descubrimientos, y ¿qué tipo de historia haríamos si no explicamos causalidades y condicionamientos?. Precisamos introducir desde el principio la historia cualitativa», BARROS, C., Mentalidad y Revuelta, ed. facsímil, Santiago de Compostela, 1988.

"CUADERNOS DE ESTUDIOS GALLEGOS", Tomo XLVII, Fascículo 113, Santiago 2000. 
proceso, pero no mide las variables numéricamente, aunque puede determinar que hay cambios de valor ${ }^{34}$.

A lo largo de todo el siglo XX, la prensa se ha convertido en una fuente documental de mucho uso para el historiador ${ }^{35}$, aunque escolásticamente se valore mejor aquello que es más lejano en el tiempo que la Historia inmediata. Esta última, está en manos de periodistas y aficionados a la Historia, hay pocos historiadores profesionales dedicados a ella. Los historiadores se deben dar cuenta de que lo que está sucediendo en el momento es ya historia, el periodista o el aficionado sólo es un medio, que intencionadamente o no, puede variar la noticia según unas u otras creencias. En general la figura del periodista no difiere mucho de los escribanos de la Edad Media o Moderna. Lo que se debe tener presente cuando se trabaja con prensa es la línea ideológica que sigue el diario en cuestión. En este caso, el diario El País sigue una línea «social-liberal».

Del aspecto formal del texto se puede deducir que el volumen real de la traducción no se corresponde estrictamente con el número de párrafos traducidos (un 44.8\%), sino con el de líneas traducidas. A pesar de esto, se han escogido como unidad los párrafos y no las líneas, porque es más operativo, tanto para cuantificar como para explicar. Es significativo que no se hubiera convertido al idioma cervantino ninguna de las dieciocho notas a pie de página, la nota aclaratoria inicial y, lo que es peor, no se respetó la estructura del texto en cuanto a los cinco epígrafes. Lo mismo sucedió con la «h» minúscula de la palabra «historia» en el título del artículo. Todos estos elementos contribuyeron considerablemente a crear confusión.

\footnotetext{
${ }^{34}$ ARÓSTEGUI, Julio, La investigación histórica: teoría y métódo, p. 360. Aróstegui también señala que «las técnicas cualitativas serían aquellas que no aspiran a medir en la construcción de datos. Su aspiración es, por tanto, la de clasificar, tipologizar, reunir los datos, pues, en función de su cualidad, de su carácter» (ARÓSTEGUI 1995: 359360).

${ }^{35}$ Eric Hobsbawm señalaba la importancia de la prensa para elaborar su libro Historia del siglo $X X$; allí argüía: «a medida que el historiador del siglo $\mathrm{XX}$ se aproxima al presente depende cada vez más de dos tipos de fuentes: la prensa diaria y las publicaciones y los informes periódicos, por un lado, y los estudios económicos y de otro tipo, las compilaciones estadísticas y otras publicaciones de los gobiernos nacionales y de las instituciones internacionales, por otro»; HOBSBAWM, Eric, Historia del siglo XX, trad. Juan Faci, Jordi Ainaud y Carme Castells, Crítica, Barcelona, 1995 (Age of extremes the short twentieth century, 1914- 1991, Pantheon books, 1994).
}

"CUADERNOS DE ESTUDIOS GALLEGOS", Tomo XLVII, Fascículo 113, Santiago 2000. 
En líneas generales, la traducción era algo inferior a ese $48 \%$ que se señala en el análisis por párrafos. No se debe olvidar que 11 de los párrafos eran traducidos parcialmente. La introducción y el epígrafe « $\mathrm{V}$ » fueron los únicos apartados traducidos íntegramente. El subtítulo «I» quedaba totalmente inédito. Así, en la versión del diario El País no se hacía referencia al reconocimiento por parte de Fukuyama de la falta de originalidad de «el fin de la Historia»; ni se daba a conocer la figura de Kojève, su importancia en el pensamiento de Fukuyama y su evolución profesional.

Del epígrafe «II» sólo se plasmaban unas líneas del original. Esas pocas palabras no reflejaban la explicación del idealismo (la relación entre lo real y lo ideal) en Hegel, Kojève y Fukuyama; también faltaba la postura de la escuela del materialismo determinista del Wall Street Journal; y tampoco se hacía eco del desarrollo de las actitudes ante el trabajo de los católicos y protestantes, extraídas de libro de Max Weber La ética protestante y el espíritu del capitalismo. Al dejar todas estas partes del subtítulo inéditas, la versión del diario madrileño de «el fin de la Historia» quedaba sin cimientos teóricos.

El epígrafe «III» fue un 53\% traducido en relación a sus párrafos, aunque sólo un $23.1 \%$ de los mismos fueron volcados al castellano íntegramente. Aquí se recolectaban las conclusiones finales del epígrafe sobre el fracaso del fascismo y del comunismo (en China y la U. R. S. S.) y las nuevas amenazas al régimen democrático liberal: el nacionalismo y el islamismo. En este caso se nombraba en su totalidad, pero no se explicaba casi nada; el traductor se limitó a recolectar párrafos de aquí y de allá para que las ideas generales apareciesen reflejadas en el texto.

El epígrafe «IV» presentaba el mismo problema y gran parte de la argumentación no se trasladaba a la versión española. Las interpretaciones históricas sobre las relaciones internacionales en los siglos XIX y XX hasta hoy, permanecieron vírgenes para el lector peninsular. Únicamente se transportaron a la referencia española las estimaciones sobre el futuro de las relaciones internacionales y la improbabilidad de nuevos conflictos entre los estados de «el fin de la Historia» (Fukuyama también los denomina estados poshistóricos).

En definitiva, como los porcentajes dejaban bien claro en el apartado anterior, la traducción fue incompleta, inexacta e imprecisa. De igual modo, cumplía perfectamente con la intención informativa para la que estaba

"CUADERNOS DE ESTUDIOS GALLEGOS", Tomo XLVII, Fascículo 113, Santiago 2000. 
diseñada: todo el mundo se enteró, aunque fuera de forma resumida, de las líneas de razonamiento de la tesis de «el final de la Historia». La traducción aprovechó todas las conclusiones de Fukuyama en sus partes más polémicas para enganchar al lector, pero permaneció enterrado y olvidado en la versión norteamericana todo el desarrollo del discurso histórico y teórico. Unos meses después, Fukuyama hacía pública una réplica a sus críticos en la misma revista The National Interest. Para esa ocasión, quizá por la extensión de la contestación a sus detractores, el diario El País cambiaba de estrategia y la publicaba entera. Era demasiado tarde: el original ya habían sido mutilado y no había vuelta atrás.

\section{La traducción de la revista Claves de Razón Práctica. Abril 1990}

La revista Claves de Razón Práctica ${ }^{36}$ incluía en su primer número de abril de 1990, cuatro meses después de aparecer en El País, la traducción integra del artículo ${ }^{37}$ «El final de la Historia?» en su sección documentos. Esta nueva versión en castellano, incorporaba la mudanza al español de las dieciocho notas a pie de página, la división por epígrafes respetando la original y se añadía la denominación por números para cada sección del texto. El texto consistía en doce páginas maquetadas a cuatro columnas, con las notas en el pie de la columna correspondiente. La palabra «Historia» aparecía con mayúscula en el título y la entradilla al texto, incluía parte de la nota introductoria original sobre la situación profesional de Fukuyama. Cabe destacar que, excepto en un párrafo del epígrafe «II» correspondiente a la explicación de Max Weber, la traducción es fiel en cuanto a la unidad y presentación de los párrafos.

\footnotetext{
${ }^{36}$ Quiero hacer constar mi agradecimiento personal a la Promotora General de Revistas, s. a. (PROGRESA), perteneciente al grupo PRISA y editora de la revista Claves de Razón Práctica. Debido a la dificultad para encontrar el primer número de Claves, agotado en la editora y ausente de las bibliotecas, me puse en contacto directamente con ellos y desde el primer momento mostraron una total disposición en ayudarme. No dudaron en mandarme desinteresadamente y con la mayor de las diligencias las fotocopias correspondientes al artículo de Fukuyama.

${ }^{37}$ En Sudamérica se hizo una traducción completa del artículo: FUKUYAMA, Francis, «¿El fin de la Historia?», El Gallo ilustrado (semanario de El día de México), 29 de abril de 1990. Este mismo artículo fue reproducido en la revista Tareas, Panamá, $\mathrm{n}^{\circ} 75$, mayo-agosto 1990, pp. 83-112. Exactamente igual sucedió en Alemania donde la referencia exacta fue: FUKUYAMA, F., «Ende der Geschichte?», Europäische Rundschau, $\mathrm{n}^{\circ} 17,1989$, pp. 16 y ss.
}

\footnotetext{
"CUADERnOS DE ESTUdios GALLEGOS", Tomo XLVII, Fascículo 113, Santiago 2000.
} 
Pese a contener gran parte del texto original, la traducción de la revista Claves no respetaba las palabras en cursiva y entre comillas del original. En ocasiones, las palabras entrecomilladas del original se volvían normales en la traducción (por ejemplo, «material» figuraba como material y «superstructure» como superestructura en la página 87). En otras palabras o grupos de palabras, las comillas se respetaban («peace» y «end of ideology» (p. 3) seguían como «paz» y «fin de la ideología» en la página 85).

Otros vocablos figuraban en la versión norteamericana entre comillas y en la traducción se hallaban en cursiva. (Éste es el caso de «natural» (p. 4) por naturales en la página 86 de la revista Claves; "contradictions»" (pp. $5,8,14$ ) se transformaba en contradicciones en las páginas 86,89 y 93). Un cambio muy común en la traducción consistía en eliminar gran parte de las cursivas de la original (Western idea (p. 3) aparecía en el artículo de Claves como idea occcidental (p. 85). A pesar de estos cambios, en otras oportunidades las cursivas se mantenían. Debido a que muchas de ellas pertenecían a revistas o títulos de libros (Foreign Affairs).

Resulta difícil dilucidar el criterio seguido por los responsables de la revista Claves para eliminar comillas o cursivas o cambiar unas por otras. Y si se acude a ejemplos concretos la confusión crece más: el término marxista «superestructura» aparecía en el original entrecomillado; en la traducción figuraba una vez en letra normal y otra en cursivas (ambas en la página 87). Otro ejemplo correspondía a la palabra «people» ${ }^{38}$. El original se ofrecía entrecomillado y en la traducción una vez en cursivas ( $p$. 89), y otra entre comillas (p. 92). ¿Cuál es el juicio empleado para entrecomillar o poner cursivas?. Agustín García Calvo, sin todavía ojear el artículo de The National Interest, razonaba que el uso de comillas o cursi-

\footnotetext{
${ }^{38} \mathrm{La}$ acotación sobre la palabra «people» pertenece a Agustín García Calvo en: GARCÍA CALVO, Agustín, "Tras el fin de la Historia», Claves de Razón Práctica, $\mathrm{n}^{\circ}$ 6, octubre 1990, p. 11. García Calvo criticaba en la página 8 la aplicación del término «pueblo». Fukuyama relacionaba la palabra «pueblo» con la reducción de la gente a masas que habían hecho el fascismo y el socialismo comunista. Fukuyama operaba para la democracia liberal con individuos bien definidos cada uno y en conjunto, donde el colectivo no impedía la identidad perfecta de cada uno. García Calvo decía que Fukuyama hacía la misma idealización que el fascismo y el comunismo, pero de manera más hábil y potente; sólo cambiaba la palabra «pueblo» por la de «hombre».
}

"CUADERNOS DE ESTUDIOS GALLEGOS", Tomo XLVII, Fascículo 113, Santiago 2000. 
vas equivalía en intención ${ }^{39}$. Sin quitarle la razón a García Calvo, más exacto es pensar que el traductor eliminó las cursivas y las comillas del original cuando eran palabras que aparentemente no tenían un significado especial, como en «pueblo», «material» o disminuir. De la misma manera, conservó muchos de los entrecomillados correspondientes a grupos de palabras como «Nuevo Pensamiento»o «legalidad burguesa». La conservación de las cursivas era más evidente porque reflejaba títulos de libros o instituciones. Y, por último, donde sí parece tener razón García Calvo, es en los cambios de comillas por cursivas; ahí hay si cabe pensar en una equivalencia de significados. De todas maneras, una buena claridad y transcripción de los términos, es fundamental para una comprensión perfecta del texto. Como se vio en este caso, pese a la meticulosidad de la traducción, muchos de estos aspectos formales llevan a confusión ya que de su entrecomillado o forma de letra dependen muchas interpretaciones. De hecho García Calvo juzga la traducción como "poco satisfactoria» ${ }^{40}$.

La traducción ofrecía algunos errores. «La batalla de Jena» era traducida por la «batalla de Iena» en las tres veces que aparecía en el texto (pp. 86 y 87). Un desacierto mayor se manifestaba en la fecha de la muerte del filósofo francés de origen ruso Alexandre Kojève. La traducción aseguraba que falleció en 1988, cuando el verdadero año de su expiración ocurrió en $1968^{41}$. Además de estas equivocaciones, había un olvido que reflejaba la actitud profesional y política de Fukuyama, que, de alguna manera, intuía la polémica que iba a generar el artículo. El olvido correspondía a las cuatro últimas líneas de la nota introductoria sobre el cargo profesional de Fukuyama. Como quedó escrito anteriormente, parte de esa nota iba en la entradilla de la revista Claves, pero se olvidaron de lo siguiente: (especialmente son significativas las tres últimas líneas):

${ }^{39}$ GARCÍA CALVO, Agustín, «Tras el fin de la Historia», p. 8. En la página 11 afirmaba haber recibido el original «después de escrito el artículo, y ya en pruebas de imprenta».

${ }^{40}$ GARCÍA CALVO, Agustín, "Tras el fin de la Historia», p. 11.

${ }^{41}$ «Kojève abandonó la actividad docente después de la guerra y dedicó el resto de su vida trabajando como un burócrata en la Comunidad Europea hasta su muerte en 1968», FUKUYAMA, F., «The end of History?», p. 5. Kojéve falleció súbitamente el día 4 de junio de 1968 en una conferencia sobre el mercado común; AUFRET, Dominique, La philosophie, l'Etat, la fin de l'Histoire, Bernard Grasset, París, 1990, p. 414.

"CUADERNOS DE ESTUDIOS GALLEGOS", Tomo XLVII, Fascículo 113, Santiago 2000. 
"El autor le gustaría dar las gracias a el Olin Center y a Nathan Tarcov y Allan Bloom por su apoyo en esto y su gran empeño inicial. Las opiniones expresadas en este artículo no reflejan las opiniones de la RAND Corporation ni de ninguna agencia del gobierno de los Estados unidos» $\rangle^{42}$.

Otras erratas menores fueron: la eliminación de siglas como NICs (Newly Industrializing Countries) que debería aparecer en la página 90, en referencia a Asia, también la desaparición de las mayúsculas, como en vecinos Asiáticos (p. 90). Por su parte, Agustín García Calvo le endosaba un error más al traductor ${ }^{43}$. Se trataba de la palabra «existirá», que aparecía en el último párrafo del artículo, que preconizaba: «En la era poshistórica no existirá ni arte ni filosofía...». Para García Calvo el «existirá» había que colgárselo al traductor. En efecto, en el original se utilizaba el tiempo futuro «there will be», pero no el verbo «existir»; la traducción correcta era «habrá $\rangle^{44}$. A pesar del error, el intercambio del verbo «haber» por el de «existir» no tenía tanta trascendencia como pretendía García Calvo, pues los dos verbos eran, en ese caso, sinónimos.

La revista Claves, cumplió con la labor de traducir en su totalidad el artículo, aunque fuera con algunas erratas y, casi un año después de su publicación en inglés. En la fecha que se publicó el artículo en Claves, Fukuyama ya había contestado a todos sus críticos en otro artículo correspondiente al número de invierno de 1989/90 de la revista National Interest.

La difusión de esta traslación de Claves fue mucho menor que la del diario El País. La incidencia popular de éste último y la repercusión más especializada de Claves actuaron como factores determinantes en el conocimiento de la tesis. En relación a los lectores de revistas como

\footnotetext{
${ }^{42}$ FUKUYAMA, F., «The end of History?», nota introductoria, p. 3.

${ }^{43}$ GARCÍA CALVO, Agustín, "Tras el fin de la Historia», p. 11.

44 «Habrá» también se encontraba en la traducción sudamericana de «The end of History?», FUKUYAMA, F., «El fin de la Historia», Tareas, Panamá, 29 abril, 1990, p. 109. En el mundo anglosajón fue reproducido en Time, Newsweek o Washington Post, entre otros.
}

"CUADERNOS DE ESTUdIOS GALLEGOS", Tomo XLVII, Fascículo 113, Santiago 2000. 
Claves $^{45}$ o The National Interest, García Calvo indicaba que sus lectores (entre los que se incluía) pertenecían a la élite de los «medios y altos ejecutivos» ${ }^{46}$.

\section{La traducción del diario $A B C$. Noviembre 1989}

Ningún crítico $^{47}$ han citado la divulgación del artículo «El fin de la Historia» en el diario monárquico y conservador $A B C$. Seguramente se haya debido a que sólo aparecieron textualmente unos breves párrafos del mismo y que llegó a los lectores tres meses después del de el diario El País. Juan Pedro Quiñonero ${ }^{48}$, el corresponsal de $A B C$ en Francia, exponía en dos páginas de una forma muy documentada, cómo la revista liberal Commentaire (fundada por Raymond Aron) había difundido «el fin de la Historia» en Francia. También explicaba, con sus palabras, el artículo de Fukuyama, intercalando diferentes párrafos del artículo original. Evidentemente no guardaba ninguna rigurosidad formal en cuanto al término Historia con minúscula o con mayúscula, ni en los demás aspectos analizados para el caso del diario El País. «He intentado resumir, mal que bien, muy sumariamente, un debate filosófico e histórico que exigirá nuevas y renovadas aproximaciones ${ }^{49}$, reconocía Quiñonero en uno de los pasajes. El artículo estaba acompañado por una caricatura de Marx desquebrajándose.

El resumen de Quiñonero iba acompañado de un artículo del conservador Dalmacio $\mathrm{Negro}^{50}$ y otro del marxista Gustavo Bueno ${ }^{51}$. Con la

${ }^{45}$ Claves se seguiría ocupando de «el fin de la Historia» en: LAMO DE ESPINOSA, Emilio, «El estado universal democrático», Claves de razón práctica, $\mathrm{n}^{\circ} 9$, enero-febrero 1991, pp. 38-46. G. SUÁREZ, Alfonso, «¿Réquiem por la Historia?. Examen crítico de la tesis de Fukuyama», Claves de razón práctica, n 32, mayo 1993, pp. 56- 60. HALPERING DONGHI, Tulio, «Promesa y paradoja en el triunfo de la democracia», Claves de razón práctica, $\mathrm{n}^{\circ} 21$, abril 1992, pp. 2-10.

${ }^{46}$ GARCÍA CALVO, Agustín, "Tras el fin de la Historia», p. 8.

${ }^{47}$ Ninguno de los críticos o comentaristas de «el fin de la Historia» que yo he consultado citan ningún artículo del diario $A B C$.

${ }^{48}$ QUIÑONERO, Juan Pedro, «El marxismo, puesto contra el muro: ¿fin de la historia?», $A B C, 26$ de noviembre de 1989, pp. 56-57.

${ }^{49}$ QUIÑONERO, Juan Pedro, «El marxismo, puesto contra el muro: ¿fin de la historia?», p. 57.

${ }^{50}$ NEGRO, Dalmacio, «La muerte de la utopía antipolítica», $A B C, 26$ de noviembre de 1989 , pp. 56 y 57.

${ }^{51}$ BUENO, Gustavo, «¿Fin de la Historia? Crisis del Este y crisis del marxismo», $A B C, 26$ de noviembre de 1989 , p. 58 .

"CUADERNOS DE ESTUDIOS GALLEGOS", Tomo XLVII, Fascículo 113, Santiago 2000. 
inclusión de estos dos trabajos, el periódico dirigido en aquel momento por Luis MaríaAnson, trataba de continuar el debate que ya habían iniciado en las «Terceras» de ese mismo periódico Francisco Ayala ${ }^{52}$ y Julián Marías $^{53}$. La discusión la continuaron el propio Quiñonero ${ }^{54}$ dos días más tarde, José María Carrascal en dos entregas ${ }^{55}$, Alejandro Muñoz Alonso ${ }^{56}$ y Federico Jiménez Losantos ${ }^{57}$.

La difusión de «El fin de la Historia» por el diario $A B C$ fue una divulgación más centrada en el debate y en las opiniones de sus comentaristas. El texto original, así como las notas a pie de página del mismo, no les interesaban. Dejaban en manos de su corresponsal la exposición de las ideas de Fukuyama. Lo sustantivo de la labor del $A B C$ era que informaba que el artículo de Fukuyama existía y, modestamente, ofrecían un resumen del mismo. Claro, casi tres meses después del diario El País.

La traducción española de «A reply to my critics». Diciembre 1989

El diario El País, se encargó , una vez más, de la divulgación del escrito de Fukuyama en España ${ }^{58}$. Apenas veintiún días después de la publicación del original, los lectores del periódico madrileño ya lo podían ojear. El artículo estaba traducido en su totalidad. Comprendía cuatro hojas, e incluía las siete notas a pie de página del original.

Con respecto a la versión norteamericana, se alteraba el título añadiéndole un subtítulo: «El fin de la historia no significa el fin de los acontecimientos del mundo». De igual modo, se agregaban, seguramente por ra-

${ }^{52}$ AYALA, Francisco, «Postrimerías de la Historia», $A B C, 18$ de noviembre de 1989, p. 3.

${ }^{53}$ MARÍAS, Julián, «Silencio», $A B C, 24$ de noviembre de 1989, p. 3.

${ }^{54}$ QUIÑONERO, Juan Pedro, «El fin de la Historia», $A B C, 28$ de noviembre de 1989, pp. 20-21.

${ }^{55}$ CARRASCAL, José María, «El fin de la Historia», $A B C, 7$ de diciembre de 1989, p. 18.; CARRASCAL, José María, «Fin de la Historia 2», $A B C, 13$ de diciembre de 1989, p. 17.

${ }^{56}$ MUÑOZ ALONSO, Alejandro, «Sobre el fin de la Historia», $A B C, 28$ de diciembre de 1989 , p. 3.

${ }^{57}$ JIMÉNEZ LOSANTOS, Federico, «¿El fin de las ideologías?», $A B C, 29$ de diciembre de 1989, p. 16.

${ }^{58}$ FUKUYAMA, Francis, «Respuesta a mis críticos», trad. Leopoldo Rodríguez Regueira, "Temas de nuestra época», El País, 21 diciembre, 1989, pp. 3-6. En el Reino Unido se publicó en The Guardian Weekly, 31 diciembre, 1989, p. 15.

"CUADERNOS DE ESTUdIOS GALLEGOS", Tomo XLVII. Fascículo 113, Santiago 2000. 
zones de estilo del rotativo, dos subtítulos: «Éramos hegelianos» $\mathrm{y}$ «Falso menosprecio» en la página 4. Para ilustrar el texto se utilizaron diversas fotocomposiciones carentes de interés para este análisis. Acompañando a la respuesta de Fukuyama, se insertaban las replicas del lingüista Noam Chomsky; el subdirector de la sección de internacional de The Independent, Robert Cotrell; el historiador de las doctrinas económicas, Pedro Schwartz; los escritores Vicente Urbistondo y Artemio Baigorri; y el sociólogo Ludolfo Paramio ${ }^{59}$.

Leopoldo Rodríguez Regueira trasladó el artículo del inglés al castellano, en una traducción demasiado poblada de imprecisiones y errores. El desliz más importante figuraba en la página 4, donde se podía leer: «Un historiador profesional puede hablarnos de la casualidad en la historia...». En el original la palabra «casualidad» era en realidad «causality» (p. 22), cuyo significado correcto es «causalidad».

Otro gazapo de interés, ocurría al convertir una frase exclamativa en una enunciativa. En la página 3 de la traducción estaba escrito: «; pero no tener tiempo de leer completo un artículo de dieciséis páginas...». En esa frase, el punto y coma inicial era un punto y seguido en la versión norteamericana (p. 21 del original); y los puntos suspensivos correspondían a una exclamación (p. 21 de The National Interest). Igual problema, pero de menor importancia, sucedía con el aforismo alemán «Wahrheit statt Mehrheit», sin admiraciones en la página 3 de la traducción de El País y con ellas en la página 21 de la revista estadounidense.

En otro sentido, en la versión española, se anulaban las dos únicas veces que Fukuyama utilizaba la «H» mayúscula. La primera competía a una mención del artículo primigenio «The end of History?» con «h» minúscula (p. 3 traducción y 21 del original); la segunda «h» minúscula,

\footnotetext{
${ }^{59}$ CHOMSKY, Noam, «El comienzo de la historia», trad. C. Scavino, en «Temas de nuestra época», El Pais, 21 diciembre, 1989, pp. 8-9; COTTRELL, Robert, «Europa en el año 2000», trad. Carmen Viamonte, en "Temas de nuestra época», El País, 21 de diciembre, 1989, pp. 10; SCHWARTZ, Pedro, «La historia no acaba aquí», en «Temas de nuestra época», El País, 21 de diciembre, 1989, pp. 12- 13; URBISTONDO, Vicente, «El tedio implacable», en «Temas de nuestra época», El País, 21 de diciembre, 1989, pp. 1314; BAIGORRI, Artemio, «La trampa Fukuyama», en «Temas de nuestra época», El País, 21 de diciembre, 1989, pp. 15; PARAMIO, Ludolfo, «El fin de la prehistoria», en «Temas de nuestra época», El País, 21 de diciembre, 1989, pp. 16.
} 
afectaba a la definición de «Historia» de Hegel (p. 3 El País y 22 de The National Interest).

En cuanto a comillas y cursivas no había ningún problema. Las comillas se utilizaban para frases hechas y referencias literales de otros artículos o comentarios y las cursivas se empleaban para distinguir los usos diferentes de una palabra, títulos de libro y artículos. Todo cumpliendo las premisas e indicaciones contenidas en el manual de estilo de El País.

\section{El libro The end of History and the last man. 1992}

Casi tres años después aparecía una ampliación del artículo de 1989 «The end of History?» en forma de libro que Fukuyama tituló The end of History and the last man ${ }^{60}$.

Formalmente las diferencias entre la versión norteamericana y española comienzan en la portada. El libro anglosajón no llevaba impreso el dibujo de Leonardo Da Vinci ni presentaba la foto de Fukuyama con una breve reseña personal. Además, las notas aparecían en la versión original al final del libro y en la versión en castellano a pie de página. Tampoco estaba incluido en la versión original la pequeña presentación de la contraportada. E incluso la encuadernación variaba y las endebles pastas que ofrecía el libro de la colección «documento» de la editorial Planeta se convertían en robustas en la edición de Free Press (aunque también había una versión rústica del libro). Asimismo, las 418 páginas de la primera versión pasaron a 474 en la castellana, en parte debido al mayor tamaño de la obra norteamericana. Otra diferencia correspondía a las citas de autores, figuraban separadas del texto en el original e inmerso en él en la traducción.

Dentro de las diferencias de contenido entre los dos libros, la versión castellana reproducía numerosos errores a lo largo del libro. Los más importantes hacían referencia al término «Historia» Todas las ocasiones en las que en la versión norteamericana estaba escrito «History» (con «H» mayúscula) fueron traducidos por «historia» (con «h» minúscula). Esto llevaba al equívoco en la traducción española de no saber cuando se está

${ }^{60}$ FUKUYAMA, Francis, The end of History and the last man, The Free Press, New York, 1992. Versión española El final de la Historia y el último hombre, Planeta, Barcelona, 1992.

"CUADERNOS DE ESTUDIOS GALLEGOS", Tomo XLVII, Fascículo 113, Santiago 2000. 
refiriendo a la Historia como evolución ideológica de la humanidad o cuando se refería a la historia como sucesión formal de acontecimientos. Exactamente lo mismo sucede con el término «Universal History», invariablemente con mayúsculas en la versión original y traducido inalterablemente como «historia universal» (salvo en el índice).

A pesar de que no es objeto de este trabajo analizar filológicamente en profundidad la traducción, es necesario tener en cuenta algunas consideraciones al respecto. En muchas ocasiones, palabras que se hallaban con cursivas en la versión original se volvían normales (The great illusion en la p. 5 del original, ideal en la p. xi, Alawi en la p. 216, principle of democracy en p. 17, Movimiento das Forces Armadas (MFA) en la p. 18, raison d'être en la p. 20, perestroika en la p. 29, bourgeois en las pp. 160 y 180, anger en la p. 164, Jodo Shinshu en la p. 227 o en masse en la p. 267.

En otras ocasiones, palabras que estaban en letra normal aparecen en cursiva: apartheid en la p. 52 y 53 de la traducción o hippie en la p. 132 de la versión castellana (en este caso es justificable por el origen no español de la palabra). Otras veces se suprimían las comillas como en «mechanism» ('mecanismo' en la p. 128 de la traducción) e «historist» ('historicista' en la p. 200 de la versión en castellano). También se añadían comillas como es el caso de «hombre económico» en la p. 197 de la traducción. $\mathrm{Y}$ en algunas oportunidades, hay palabras que a veces permanecían como cursivas y otras pasan a normales como la palabra dependencistas (dependentistas); se conservaba en cursivas en la edición castellana en la $p$. 153 y se varía en normal en las pp. 153 y 154 . Hay otros casos donde se eliminaban palabras en mayúsculas como en el caso de "LIBERTY» y «FREEDOM» de la p. 148 del original.

Otros cambios eran debidos a cuestiones de estilo. Es el caso de la persistencia en la versión norteamericana de las mayúsculas para «Left» y «Right» (entendidas como derecha e izquierda políticas), para los gentilicios como «German», «Austrian» o «Russian», en referencia a las partes del libro («Part II», «Part I»...), para los meses del año, o en relación a los títulos de los libros y artículos donde todas las primeras letras de palabras están en mayúsculas. En castellano todo esto aparecía en minúsculas salvo algunos de los títulos de libros. No obstante, la palabra «state» siempre se traducía como «Estado».

Error de mayor consideración fue el referente a la tabla «Liberal Democracies Worldwide» (Las democracias liberales en el mundo), basada

"CUADERNOS DE ESTUdIOS GALLEGOS", Tomo XLVII, Fascículo 113, Santiago 2000. 
en una estadística de Michael Doyle, que aparecía en el original en las páginas 49 y 50 y en la traducción en castellano en las hojas 86 y 87 . Mientras en la versión anglosajona se contabilizaban 61 democracias en 1990; en la traducción publicada por la editorial Planeta la cifra aumentaba hasta 62 ya que añadía, sin ninguna razón, a Yugoslavia. Por lo tanto, la cifra correcta es 61 democracias estables en 1990.

Igualmente, en el capítulo cinco «La idea de una Historia Universal» cuando Fukuyama se estaba refiriendo a Hegel y lo nombra como «the first historicist philosopher»-el primer filósofo historicista- (p. 62) era volcado al castellano como «el primer filósofo histórico» (p. 102).

Pese a todo, los errores se incrementaban en las notas, sobre todo en los primeros capítulos:

En el capítulo uno aparecía un desliz en la nota 13 donde Fukuyama citaba la obra de Jean François Revel How democracies perish ${ }^{61}$. En castellano, el How se convertía en Why.

En el capítulo tres también en la nota 13 se suprimía en la versión castellana el título del artículo de Aleksandr Solzhenitsyn «How are to restructure Russia», Literaturnaya Gazeta, $\mathrm{n}^{\mathrm{o}}$. 18, septiembre 18, 1990, pp. 3-662. En la nota 1 se apostillaba el título «La abeja y el ideal comunista», cuando no figuraba en la original. La nota quedaba así: En Yu. Afanaseyev, ed.., Inogo ne dano, Moscú, Progress, 1989, p. $510^{63}$.

En el capítulo cuatro ocurrió posiblemente un fallo informático y salieron parte de las notas equivocadas ${ }^{64}$ :

En la nota 1 figuraba en el libro español «Dokumente, p. 352», cuando en realidad debiera aparecer: Dokumente zu Hegels entwicklung, ed. J. Hoffmeister, Stuttgar, 1936, p. $352^{65}$.

En la nota 2 no se debe tener en consideración el texto que aparece en la nota de la versión castellana. Debiera poder leerse: Una visión de este cambio es dado, inter alia, en Sylvia Nasar, «Third world embracing re-

\footnotetext{
${ }^{61}$ FUKUYAMA, F., The end of History and the last man, p. 342

${ }^{62}$ FUKUYAMA, F., The end of History and the last man, p. 346.

${ }^{63}$ FUKUYAMA, F., The end of History and the last man, p. 345.

${ }^{64}$ Las notas se atienen a la metodología seguida en este trabajo para citar y no sigue la forma empleada por Fukuyama en su libro original ni por el traductor en castellano.

${ }^{65}$ FUKUYAMA, F., The end'of History and the last man, p. 347.
} 
forms to encourage economic groeth», New York Times, 8 de Julio, 1991, p. $\mathrm{a} 1^{66}$.

La nota 3 también está totalmente equivocada y, según la versión original norteamericana, tiene que leerse: Para un informe del repensamiento de la legitimidad de las dictaduras revolucionarias que han tenido lugar en Latinoamérica en la década pasada, ver Robert Barros, «The left and democracy: recent debates in Latin America», Telos, 68, 1986, 49-70. Para un ejemplo de la confusión en la que los sucesos en el Este de Europa han llevado a la izquierda, ver André gunder Frank, «Revolution in Eastern Europe: lessons for democratic social movements (and socialist)», Third World Quartely 12, no 2, abril 1990, pp. 36-5267.

La nota 4 presentaba un texto en castellano totalmente errado. Se debe sustituir por la simple referencia bibliográfica: James Bryce, Modern Democracies, vol. 1, Macmillan, New York, 1931, pp. 53-54 ${ }^{68}$.

La nota 5 ofrecía los mismo signos de equivocación que las anteriores. El texto que figura allí es necesario cambiarlo por:Aceptando los requisitos de Schumpeter para la definición de las democracias del siglo XVIII, podemos decir con él que la democracia es «la libre competencia entre aspirantes a dirigentes por el voto de los electores». Joseph Schumpeter, Capitalism, Socialism and Democracy, New York, Harper Brothers, 1950, p. 284. Véase también la discusión acerca de las definiciones de democracia en Samuel Huntington, «Will more countries become democratic?», Political Science Quartely 99, $n^{\circ} 2$, verano 1994, 193-21869.

La nota 6 de el libro The end of History and the last man es la que aparecía como nota 4 en la versión española, concretamente en la página 79. El texto es: La extensión del derecho de voto fue un proceso gradual en la mayoría de las democracias incluido Inglaterra y los Estados Unidos; muchas democracias contemporáneas no alcanzaron el voto universal hasta bien avanzado el siglo actual, y sin embargo se podía hablar de ellas como democracias antes de llegar a este punto. Véase Bryce, vol. I, 1931, pp. $20-23^{70}$.

${ }^{66}$ FUKUYAMA, F., The end of History and the last man, p. 347.

${ }^{67}$ FUKUYAMA, F., The end of History and the last man, p. 347.

${ }^{68}$ FUKUYAMA, F., The end of History and the last man, p. 347.

${ }^{69}$ FUKUYAMA, F., The end of History and the last man, p. 347.

${ }^{70}$ FUKUYAMA, F., The end of History and the last man, p. 347.

"CUADERNOS DE ESTUdios GALLEGOS", Tomo XLVII, Fascículo 113, Santiago 2000. 
La nota 7 del original se correspondía con la nota 6 de la traducción: Ha habido presiones para una mayor democracia en varios países del Oriente Medio como Egipto y Jordania, siguiendo las revoluciones de la Europa del Este en 1989. Pero en esta parte del mundo, el Islam el ha sido una barrera importante para una mayor democratización. Como demostraron las elecciones de Argelia de 1990, o las de Irán un decenio antes, una mayor democracia puede no conducir a una mayor liberalización porque lleva al poder a los fundamentalistas islámicos, que aspiran a establecer algunas forma de teocracia islámica ${ }^{71}$.

La nota 12 de la versión norteamericana se cambiaba por la nota «*» en la traducción castellana. Además de esto, a la versión hispana se le añadían unas líneas que sobraban: «p. 13»; Howard Wiarda, «Toward a framework for the study of political change in the Iberian Latin tradition», World Politics, 25, enero de 1973, pp. 106- 135» ${ }^{72}$.

En la nota 13 del libro castellano, que era la 14 en la obra norteamericana tras el cambio de la nota 12 por el asterisco, debería figurar: Howard Wiarda, «Toward a framework for the study of political change in the Iberio-Latín tradition», World Politics 25, enero 1973, 106-13573.

En el capítulo 5 «La idea de una Historia Universal», había un error en la nota 39 en la referencia de las páginas del artículo de Howard Wiarda «The ethnocentrism of the Social Science [sic]: Implications for research and policy». En El final de la Historia y el último hombre venía impreso que ese artículo correspondía a las páginas 153-197 cuando era pp. 163$197^{74}$.

En la nota 23 del capítulo 9 se suprimía «Brasil» de la versión hispana en la nota: Sobre el sector público en Brasil, véase Baer, 1989, pp. 238$273^{75}$.

En el capítulo 19 «El Estado Universal y Homogéneo», se suprime en la nota 3 de la traducción al español que pertenece a la página 7 del libro de Gellner ${ }^{76}$.

\footnotetext{
${ }^{71}$ FUKUYAMA, F., The end of History and the last man, p. 347.

${ }^{72}$ FUKUYAMA, F., The end of History and the last man, p. 348.

${ }^{73}$ FUKUYAMA, F., The end of History and the last man, p. 348.

${ }^{74}$ FUKUYAMA, F., The end of History and the last man, p. 352.

${ }^{75}$ FUKUYAMA, F., The end of History and the last man, p. 359.

${ }^{76}$ FUKUYAMA, F., The end of History and the last man, p. 373.
}

"CUADERNOS DE ESTUDIOS GALLEGOS", Tomo XLVII, Fascículo 113, Santiago 2000. 
En la nota 4 del capítulo 28, se eliminaba de la monografía española al final de la nota: citado en Strauss, 1991, p. $238^{77}$. En la nota 6 de ese mismo capítulo se obvió al principio de la nota en la traducción: «El grupo de trabajo de amor propio de California.... ${ }^{78}$.

Otras erratas del libro español en las notas eran las inevitables de presentar palabras originalmente en cursiva a letra normal (Idealpolitik en la nota 2 -capítulo 26- de la p. 385 de la versión norteamericana); los fallos como reflejar «Societología» en lugar de «Sovietología» (nota 20 de la página 346 y del capítulo 3 de la edición de Free Press); o eliminación de entrecomillados como «repackager» por envoltorios (nota 5 del capítulo 3 en la página 345 del original).

Este ejercicio no se debe ver como un examen filológico del libro ya que no se trata de analizar la traducción en sí; sino de apuntalar los defectos formales de la fuente para saber que tipo de texto se está manejando y corregir posibles defectos que permitan analizar y trabajar la fuente con más objetividad.

\section{CONCLUSIÓN}

Los textos de Fukuyama que se divulgaron en España se hicieron de una forma diversa. La traducción del diario El País del artículo «The end of History?» fue la más errónea y la más consultada por los críticos. Más que una traducción se debe considerar como una forma de dar a conocer un texto a un público masivo desde un periódico «generalista». Con estas premisas no se puede pedir una rigurosidad en notas a pie de página etc., que se le exige a una revista científica. Sin embargo, en el segundo artículo «A reply to my critics» el diario progresista lograba una traducción casi perfecta. En cuanto a la difusión del otro gran diario nacional, $A B C$, se centraba más bien en el debate y sólo daba a conocer sumariamente el artículo original incluyendo la traducción directa de algunos párrafos. La divulgación que realizó la revista Claves fue la más acertada aunque a lo largo de este trabajo se señalan sus puntos débiles. Pero fue la única com-

\footnotetext{
${ }^{77}$ FUKUYAMA, F., The end of History and the last man, p. 386.

${ }^{78}$ FUKUYAMA, F., The end of History and the last man, p. 387.
} 
pleta. En cuanto al libro, El fin de la Historia y el último hombre, fue traducido con algunas erratas que se señalan en el apartado correspondiente. En referencia a la diferencia de fechas entre el original y las traducciones, todas se realizaron con una diferencia entre dos meses y siete meses del artículo de The National Interest, espacio de tiempo correcto al tratarse de un autor prácticamente desconocido.

La teoría de «el fin de la Historia» tuvo una divulgación más que correcta en España. Hubo muchas erratas de estilo e informaciones que no se difundieron pero, en general, se daban todas las pistas para recurrir a las fuentes originales. En este trabajo ha quedado patente la necesidad de traducciones rigurosas, milimétricas y cuidadas para no inducir a errores. Muchas erratas tipográficas pueden llevar a grandes equívocos, como se ha escrito en páginas anteriores. Esta divulgación hizo posible el conocimiento de la teoría en España y, lo que es más importante, fomentó el debate, algo fundamental para el avance de la disciplina de la Historia ${ }^{79}$.

${ }^{79}$ Siguiendo a Kuhn, Carlos Barros asegura que «Es preciso recuperar este espíritu inconformista, crítico, resucitar la historia-debate, para superar la crisis finisecular de la historia, y también para, después de ello, alimentar el nuevo paradigma común, aprendiendo de la historiografía pasada». Ver BARROS, Carlos, «La historia que viene», en Historia a Debate, Tomo I, Santiago de Compostela, p. 112.

"CUADERNOS DE ESTUDIOS GALLEGOS", Tomo XLVII, Fascículo 113, Santiago 2000. 